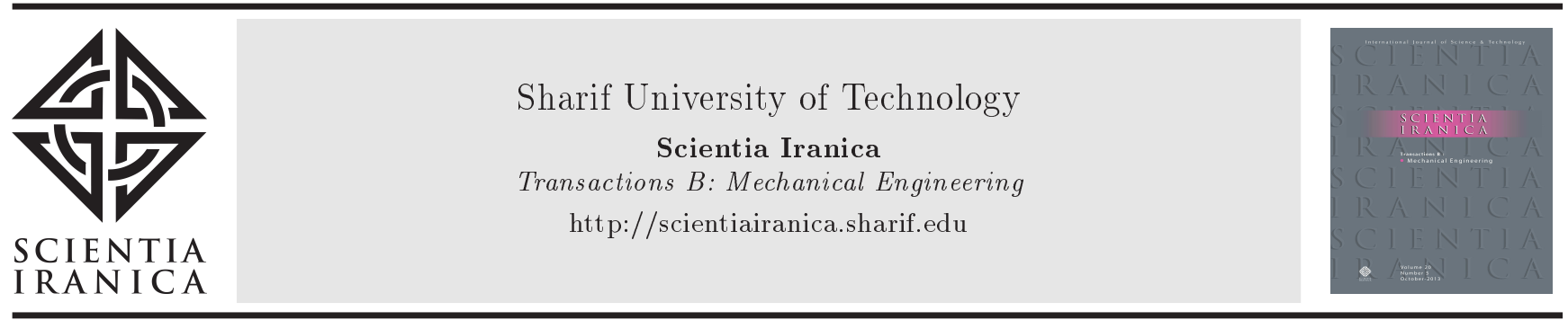

\title{
MHD peristaltic slip flow of Casson fluid and heat transfer in channel filled with a porous medium
}

\author{
O.D. Makinde ${ }^{\mathrm{a},{ }^{*}}$ and M. Gnaneswara Reddy ${ }^{\mathrm{b}}$ \\ a. Faculty of Military Science, Stellenbosch University, Private Bag X2, Saldanha 7395, South Africa. \\ b. Department of Mathematics, Acharya Nagarjuna University Campus, Ongole-523 001, India. \\ Received 12 February 2017; received in revised form 23 April 2017; accepted 16 April 2018
}
KEYWORDS
Peristaltic flow; MHD;
Casson fluid;
Heat transfer;
Porous medium;
Partial slip.

\begin{abstract}
We examined the effect of velocity slip on hydromagnetic peristaltic flow of a Casson fluid and heat transfer through an asymmetric channel fluid filled with a porous medium. The equations governing the model were obtained, simplified using long wavelength and low Reynolds number assumptions, and then tackled analytically. Numerical results for the effects of the embedded parameters on the stream function, axial velocity, pressure drop, temperature, skin friction, and Nusselt number are presented graphically and discussed in this study. It was found that the permeability parameter enhanced the size of the trapped bolus, while velocity slip diminished it. A rise in magnetic field intensity and Casson fluid parameters decreased both velocity and temperature profiles. The presented problem is of substantial importance in crude oil refinement and biomedical engineering.

(C) 2019 Sharif University of Technology. All rights reserved.
\end{abstract}

\section{Introduction}

Peristaltic motion describes the fluid transport by contraction and relaxation of an extensible channel or tube walls. It is the pumping process of fluid motion along the wave propagation route. Peristalsis plays a vital role in physiological fluids transport and can be found in several biological and biomedical systems such as gastrointestinal tract, hemolysis, bypass, esophagus, lymph, small intestine, peristaltic pump, and heartlung machines [1-7].

Moreover, studies related to complex interaction of peristaltic motion of a conducting fluid with externally imposed magnetic field will enhance the understanding of the performance of conductive physiological

\footnotetext{
*. Corresponding author. Tel.: 27-822005182; Fax: 27-21-5550775

E-mail addresses: makinded@gmail.com (O.D. Makinde); mgrmaths@gmail.com (M. Gnaneswara Reddy).
}

fluids, like blood flow in small vessels, blood pump machines, and MHD compressor cilia transport. Stud et al. [8] reported that the presence of magnetic field might enhance the arterial blood flow. Magnetic field effects on movement of erythrocytes in plasma were investigated by Srivastava and Agrawal [9]. Agrawal and Anwaruddin [10] theoretically investigated the influence of an externally imposed magnetic field on peristaltic motion of electrically conducting liquid in an equally branched stenosed channel. Their results revealed the suitability of magnetic field for the blood flow control mechanism in cardiac operations. Hydromagneticperistaltic flow has been discussed by many researchers [11-14] with a view to understanding some practical phenomena such as blood pump machine and Magnetic Resonance Imaging (MRI), which is used for diagnosis of brain, vascular diseases, and all the parts of human body. Sarkar et al. [15] presented numerical results for the influence of thermal radiation and buoyancy force on hydromagneticperistaltic motion of nanofluids in a heated asymmetric channel. The presence of surface lubrication in peristaltic fluid motion 
through microchannels or small vessels leads to velocity slip; hence, the application of no-slip condition may not be realistic for this type of flow [16-23]. Srinivas et al. [24] investigated the effects of velocity slip and wall properties on hydromagnetic peristaltic transport with heat transfer. Similar flow problem in a vertical annulus was numerically investigated by Mekheimer and Abd Elmaboud [25]. Other relevant work on the combined effects of velocity slip and magnetic field on peristaltic motion of a conducting fluid with heat transfer can be found in [26-32]. Bhatti et al. [33-40] studied the peristalsis with different aspects and conditions.

The study of non-Newtonian fluids has attracted more significant attention due to their extensive applications in applied engineering and industry, e.g., extraction of crude oil from petroleum oil products, production of syrup drugs, and production of plastic materials. Casson fluid is one of the non-Newtonian fluids with distinct features. Its rheological model, as the name suggests, was first proposed by Casson [41]. The main objective of the present study is to extend the peristaltic flow analysis of Akbar [42] to include the effects of heat transfer with magnetic field, velocity slip, porous medium, and uniform heat source. The effect of magnetic field on the flow and heat transfer in a peristaltic motion was examined by Nadeem and Akram [43]. Ijaz et al. [44] studied the effects of nanoparticles on hydromagnetic flow of non-Newtonian fluids. Srinivas and Muthuraj [45] reported the analytical solution for peristaltic flow of a Jeffrey fluid in an inclined asymmetric channel with slip. Here, model equations are obtained and solved analytically. Also, pertinent results are displayed graphically and discussed.

\section{Mathematical formulation}

Consider the motion of an incompressible, electrically conducting Casson fluid in an irregular channel filled with a saturated porous medium with heat transfer under the influence of a transversely imposed magnetic field of strength $B_{0}$. The magnetic Reynolds number is assumed to be small so that induced electric field is negligible. The flow is driven by sinusoidal wave trains propagating with constant speed $c$ over the channel walls. Figure 1 presents the physical geometry of the problem.

The expressions of wall surfaces for the retained flow in the irregular channel are:

Upper wall:

$$
Y=H_{1}(X, t)=d_{1}+a_{1} \cos \left[\frac{2 \pi}{\lambda}(X-c t)\right] .
$$

Lower wall:

$$
Y=H_{2}(X, t)=-d_{2}-b_{1} \cos \left[\frac{2 \pi}{\lambda}(X-c t)+\phi\right],
$$

where $a_{1}$ and $b_{1}$ are the amplitudes of the waves, $\lambda$ is wavelength, $d_{1}+d_{2}$ is width of the channel, $c$ is velocity of propagation, $t$ is time, $X$ is direction of wave propagation, $Y$ is perpendicular to $X$, and $\phi$ is the phase difference, which varies in the range of $0 \leq \phi \leq \pi$. In addition, $a_{1}, a_{2}, b_{1}, b_{2}$, and $\phi$ should satisfy the following condition:

$$
a_{1}^{2}+b_{1}^{2}+2 a_{1} b_{1} \cos \phi \leq\left(a_{1}+a_{2}\right)^{2} \text {. }
$$

The governing equations of mass, momentum, and heat conservation for 2D unsteady flow of incompressible fluid considering magnetic field, porous medium, and joule heating impacts are given as:

$$
\begin{aligned}
& \frac{\partial U}{\partial X}+\frac{\partial V}{\partial Y}=0 \\
& \rho\left[\frac{\partial}{\partial t}+U \frac{\partial}{\partial X}+V \frac{\partial}{\partial Y}\right] U=-\frac{\partial P}{\partial X}+\frac{\partial}{\partial X}\left(S_{X X}\right) \\
& +\frac{\partial}{\partial Y}\left(S_{X Y}\right)-\sigma B_{0}^{2} U-S_{X Y} \frac{\mu}{K} U \\
& \rho\left[\frac{\partial}{\partial t}+U \frac{\partial}{\partial X}+V \frac{\partial}{\partial Y}\right] V=-\frac{\partial P}{\partial Y}+\frac{\partial}{\partial X}\left(S_{X Y}\right) \\
& \quad+\frac{\partial}{\partial Y}\left(S_{Y Y}\right), \\
& \rho c_{p}\left(\frac{\partial T}{\partial t}+U \frac{\partial T}{\partial X}+V \frac{\partial T}{\partial Y}\right)=k\left(\frac{\partial^{2} T}{\partial X^{2}}+\frac{\partial^{2} T}{\partial Y^{2}}\right) \\
& +\nu S_{X Y}\left[2\left(\frac{\partial U}{\partial X}\right)^{2}+2\left(\frac{\partial V}{\partial X}\right)^{2}+\left(\frac{\partial U}{\partial Y}+\frac{\partial V}{\partial X}\right)^{2}\right] \\
& +\left[\sigma B_{0}^{2}+S_{X Y} \frac{\mu}{K}\right] U^{2}+Q_{0} .
\end{aligned}
$$

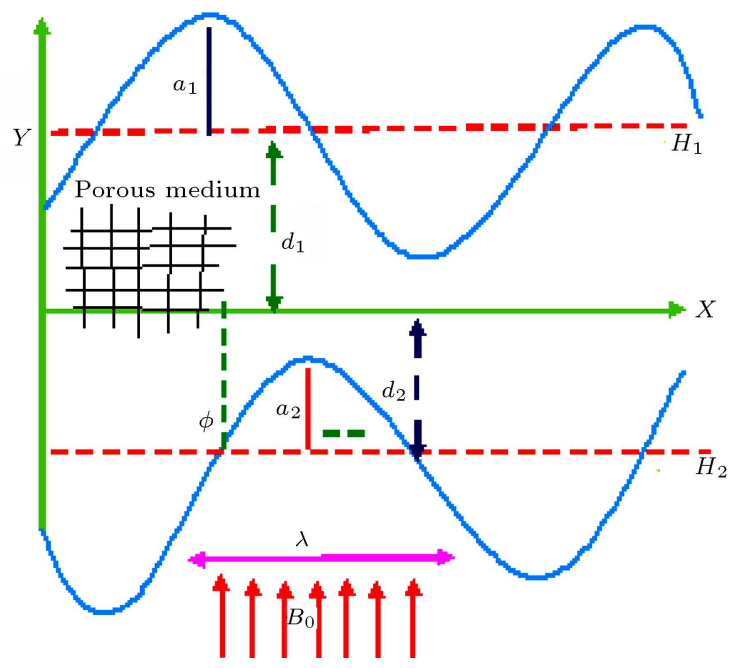

Figure 1. Geometry of the problem. 
The stress tensor for isotropic flow of a Casson fluid [41] can be expressed as:

$$
S_{i j}= \begin{cases}2 e_{i j}\left(\mu_{B}+s_{y} / \sqrt{2 \pi}\right), & \pi>\pi_{c}, \\ 2 e_{i j}\left(\mu_{B}+s_{y} / \sqrt{2 \pi}\right), & \pi<\pi_{c} .\end{cases}
$$

Here, $s_{y}$ is yield stress of the fluid, $\pi=e_{i j} e_{i j}$ is product of the component of deformation rate with itself, $e_{i j}$ is the $(i, j)$-th component of deformation rate expressed as $e_{i j}=\frac{1}{2}\left(\frac{\partial v_{i}}{\partial x_{j}}+\frac{\partial v_{j}}{\partial x_{i}}\right), \pi_{c}$ is a critical value of $\pi$ based on the non-Newtonian model, and $\mu_{B}$ is the plastic viscosity of the fluid. This model reduces to Newtonian model to vanish yield stress, i.e., $s_{y}=0$. We introduce the transformation between the wave frame $(x, y)$ and laboratory frame $(X, Y)$ in order to facilitate the analytical solutions:

$$
x=X-C t, \quad y=Y, \quad u=U-c, \quad p(x)=P(X, t) .
$$

$u$ and $v$ are the velocity components in the wave frame $(x, y)$, and $p$ and $P$ are pressure in wave and fixed frame of references, respectively. The following dimensionless variables and quantities are introduced:

$\bar{x}=\frac{2 \pi x}{\lambda}, \quad \bar{y}=\frac{y}{d_{1}}, \quad \bar{u}=\frac{u}{c}, \quad \bar{v}=\frac{v}{c}$,

$\delta=\frac{2 \pi d_{1}}{\lambda}, \quad d=\frac{d_{2}}{d_{1}}, \quad \bar{p}=\frac{2 \pi d_{1}^{2} p}{\mu c \lambda}, \quad \bar{t}=\frac{2 \pi c t}{\lambda}$,

$h_{1}=\frac{H_{1}}{d_{1}}, \quad h_{2}=\frac{H_{2}}{d_{2}}, \quad a=\frac{a_{1}}{d_{1}}, \quad b=\frac{b_{1}}{d_{1}}$,

$\operatorname{Re}=\frac{c d_{1}}{\nu}, \quad M=\sqrt{\frac{\sigma}{\mu}} B_{0} d_{1}, \quad K=\frac{k_{0}}{d^{2}}, \quad \bar{S}=\frac{S d_{1}}{\mu c}$,

$\zeta=\mu_{B} \sqrt{2 \pi_{c}} / s_{y}, \quad \mathrm{Ec}=\frac{c^{2}}{C^{\prime}\left(T_{1}-T_{0}\right)}, \quad \operatorname{Pr}=\frac{\mu c_{p}}{k}$,

$\theta=\frac{T-T_{0}}{T_{1}-T_{0}}, \quad \beta=\frac{Q_{0} d_{1}^{2}}{k\left(T_{1}-T_{0}\right)}, \quad u=\frac{\partial \psi}{\partial y}, \quad v=-\frac{\partial \psi}{\partial x}$.

Employing the low Reynolds number and long wavelength suppositions in Eqs. (4)-(7) with the aid of Eqs. (8)-(10), the dimensionless model equations for momentum and energy balance can be written as:

$$
\begin{gathered}
\left(1+\frac{1}{\varsigma}\right) \frac{\partial^{4} \psi}{\partial y^{4}}-\left[M^{2}+\left(1+\frac{1}{\varsigma}\right) \frac{1}{K}\right] \frac{\partial^{2} \psi}{\partial y^{2}}=0 \\
\frac{1}{\operatorname{Pr}} \frac{\partial^{2} \theta}{\partial y^{2}}+\operatorname{Ec}\left(1+\frac{1}{\varsigma}\right)\left(\frac{\partial^{2} \psi}{\partial y^{2}}\right)^{2} \\
+\left[M^{2}+\left(1+\frac{1}{\varsigma}\right) \frac{1}{K}\right]\left(\frac{\partial \psi}{\partial y}\right)^{2}+\beta=0 .
\end{gathered}
$$

The reduced boundary conditions for the flow in the wave frame are:

$$
\begin{aligned}
& \psi=\frac{q}{2}, \quad \frac{\partial \psi}{\partial y}+L\left(1+\frac{1}{\varsigma}\right) \frac{\partial^{2} \psi}{\partial y^{2}}=-1, \quad \theta=0 \\
& \text { at } h_{1}=1+a \cos (x) \\
& \psi=-\frac{q}{2}, \quad \frac{\partial \psi}{\partial y}-L\left(1+\frac{1}{\varsigma}\right) \frac{\partial^{2} \psi}{\partial y^{2}}=-1, \quad \theta=1 \\
& \text { at } h_{2}=-d-b \cos (2 \pi x+\phi) .
\end{aligned}
$$

Here, $\zeta, M, K, \operatorname{Pr}$, Ec, $L$, and $\beta$ are the Casson fluid parameter, Hartman number, permeability parameter, Prandtl number, Eckert number, slip parameter, and heat source parameter, respectively, and $q$ is the flux in the wave frame; $a, b, \phi$, and $d$ satisfy the relation:

$$
a^{2}+b^{2}+2 a b \cos \phi \leq(1+d)^{2} \text {. }
$$

\section{Solution to the problem}

Now, solving Eqs. (11) and (12) along the boundary conditions (13) and (14), the exact solutions to stream function and non-dimensional temperature can be obtained by Eqs. (16) and (17):

$$
\psi=F_{0}+F_{1} y+F_{2} \cosh N y+F_{3} \sinh N y .
$$

Eq. (17) is shown in Box I.

The unknowns involved in Eqs. (16) and (17) are defined in Appendix as shown in Box A.I.

The volume flux at any channel cross-section in the fixed frame is:

$$
Q=\int_{h_{2}}^{h_{1}}\left(\frac{\partial \psi}{\partial y}+1\right) d y=h_{1}-h_{2}+q
$$

$$
\theta=-\frac{1}{8 N}\left[\begin{array}{c}
16 B F_{1} F_{3} \cosh (N y)+N\left(F_{2}^{2}+F_{3}^{2}\right)\left(B+A N^{2}\right) \cosh (2 N y) \\
+\left(2\left(8 B F_{1} F_{2} \sinh (N y)+\left(\begin{array}{c}
N\left(2 C y^{2}+2 B F_{1}^{2} y^{2}-B F_{2}^{2} N^{2} y^{2}\right. \\
+B F_{3}^{2} N^{2} y^{2}+A F_{2}^{2} N^{4} y^{2}-A F_{3}^{2} N^{4} y^{2} \\
-4 c_{1}-4 y c_{2}+F_{2} F_{3}\left(B+A N^{2}\right) \sinh (2 N y)
\end{array}\right)\right)\right.
\end{array}\right] .
$$


The time-mean flow over a period $T$ is defined as:

$$
\Theta=\frac{1}{T} \int_{0}^{T} Q d t=\frac{1}{T} \int_{0}^{T}\left(q+h_{1}-h_{2}\right) d t=q+1+d .
$$

The pressure gradient is obtained from the dimensionless momentum equation for the axial velocity as:

$$
\frac{d p}{d x}=\frac{\partial}{\partial y}\left[\left(1+\frac{1}{\varsigma}\right) \frac{\partial^{2} \psi}{\partial y^{2}}-\left(M^{2}+\left(1+\frac{1}{\varsigma}\right) \frac{1}{K}\right)(\psi+1)\right]
$$

The dimensionless pressure rise $\Delta p$ is:

$$
\Delta p=\int_{0}^{1}\left(\frac{d p}{d x}\right) d x .
$$

$d p / d x$ was defined in Eq. (20). The shear stress and heat transfer rate in the walls of the channel are defined in terms of skin friction coefficient $\left(C_{f}\right)$ and Nusselt number $(\mathrm{Nu})$ as follows:

$$
\begin{aligned}
& C_{f}=\left.\left(1+\frac{1}{\varsigma}\right) \frac{\partial^{2} \psi}{\partial y^{2}}\right|_{y=h_{1}, h_{2}}, \\
& \mathrm{Nu}=-\left.\frac{\partial \theta}{\partial y}\right|_{y=h_{1}, h_{2}} .
\end{aligned}
$$

\section{Results and discussion}

In this section, we explore the influence of sundry emerging physical parameters on the quantities of interest to the present problem such as axial velocity, temperature, pressure gradient, pressure rise, and streamlines, which are examined through Figures 2-20. In the present study, the following default parameter values are adopted for numerical computations:

$$
\begin{aligned}
& \zeta=0.5, \quad M=1.0, \quad K=0.5, \quad L=0.1, \\
& \operatorname{Pr}=0.7, \quad \text { Ec }=1.0, \quad Q=1.0, \quad \beta=0.5, \\
& x=0.2, \quad a=0.3, \quad b=0.5, \quad d=1.0, \quad \phi=\pi / 6 .
\end{aligned}
$$

All graphs therefore correspond to these values unless specifically indicated on the respective graph. To study the behavior of the distribution of the axial velocity $(u)$, numerical calculations for the influence of slip parameter $(L)$, Hartmann number $(M)$, permeability parameter $(K)$, and Casson fluid parameter $(\zeta)$ are presented through Figures 2-5, respectively. Figure 2 displays the axial velocity for various values of slip parameter, $L$. It can be observed that the velocity increases with increase in $L$ near the channel walls, but the velocity field decreases at the center of the channel. The influence of Hartmann number on the axial velocity is analyzed in Figure 3 . It can be seen

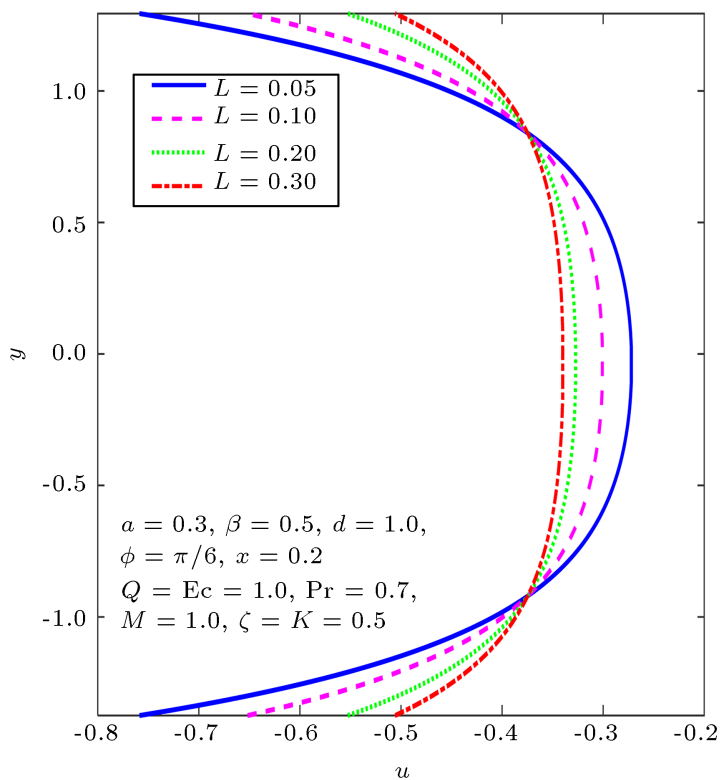

Figure 2. Impact of $L$ on $u(y)$.

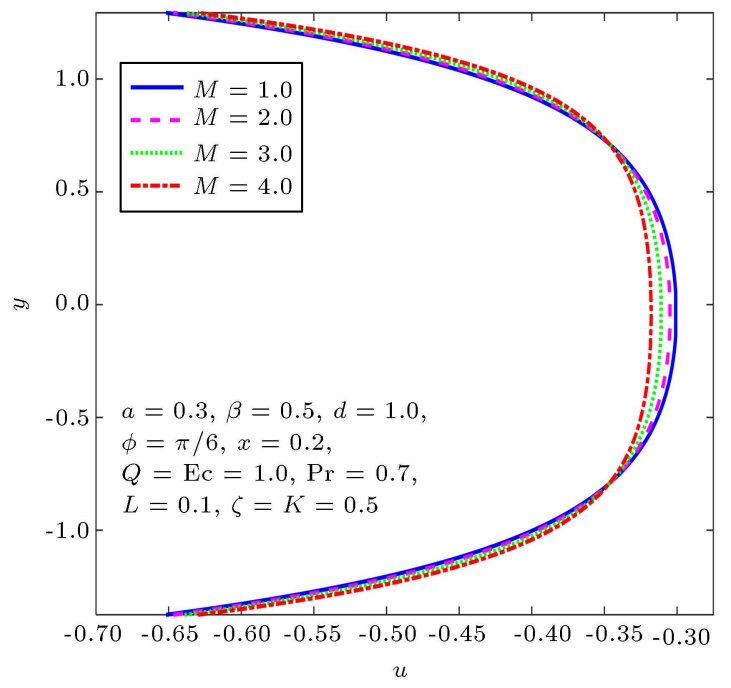

Figure 3. Impact of $M$ on $u(y)$.

that the axial velocity field increases near the channel walls and decreases in the middle of the channel. This is due to the fact that the magnetic field acts in the transverse direction to the flow and magnetic force resists the flow. Smaller increase in the maximum value of the fluid velocity when the permeability parameter is assigned higher values is evident from Figure 4. It also can be concluded that the axial velocity increases with an increase in the permeability of the porous medium in the middle of the channel. This observation is physically supported by the fact that a more permeable porous medium will provide less resistance to the fluid flow and consequently, leads to increase in the value of maximum velocity of the fluid. Figure 5 depicts that the axial velocity profile increases with an increase in Casson fluid parameter $\zeta$ at the center of the channel, 


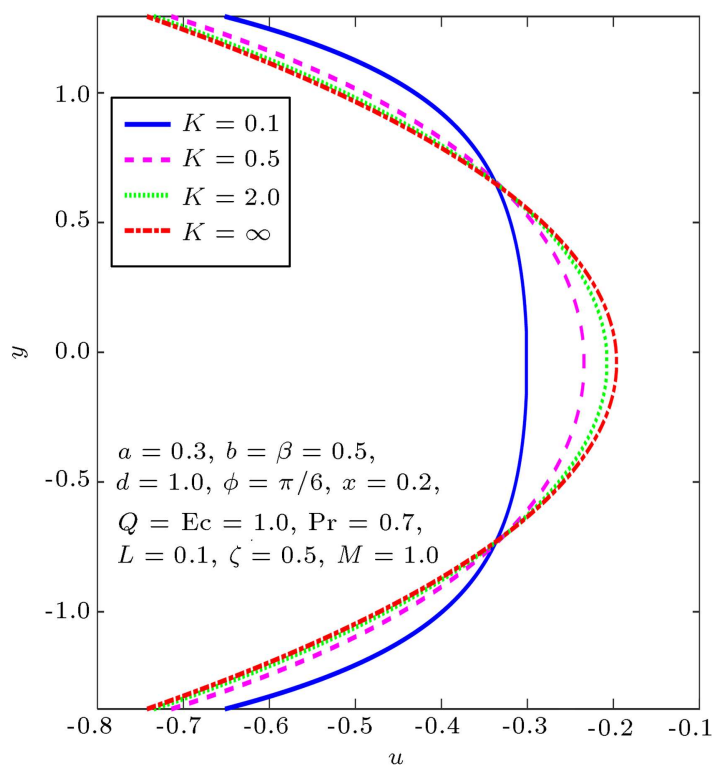

Figure 4. Impact of $K$ on $u(y)$.

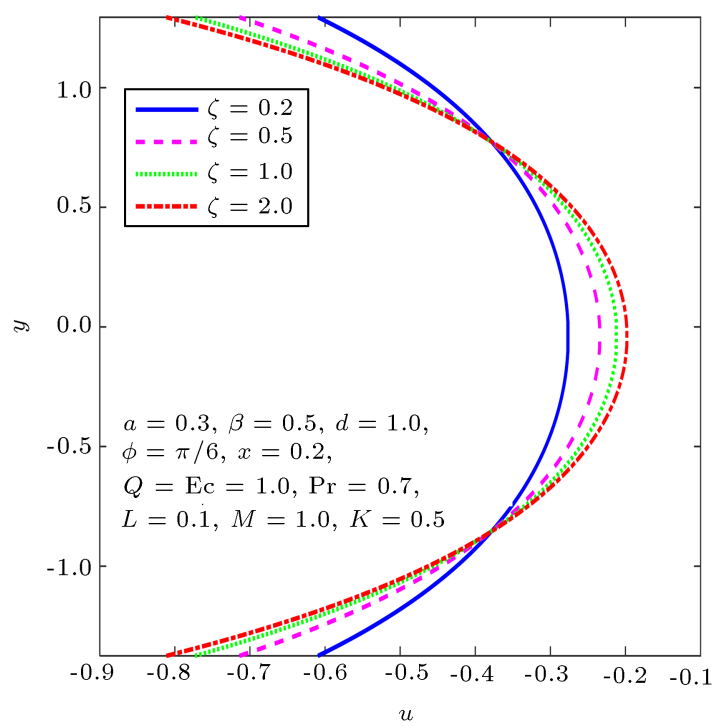

Figure 5. Impact of $\zeta$ on $u(y)$.

while the velocity field decreases near the channel walls. Our results are in good agreement with those given by Akbar [42].

The dimensionless temperature fields to perceive the behaviors of pertinent parameters like slip parameter, Hartmann number, permeability parameter, Casson fluid, Prandtl number, and heat source $\beta$ are plotted in Figures 6-9. The variation of temperature profile with various values of Hartmann number, $M$, is depicted in Figure 6. It can be concluded from Figure 6 that the temperature decreases with an increase in Hartmann number, $M$. Figure 7 displays the influence of permeability parameter on the temperature distribution. From this figure, a small decrease in the fluid temperature can be found with increase in the

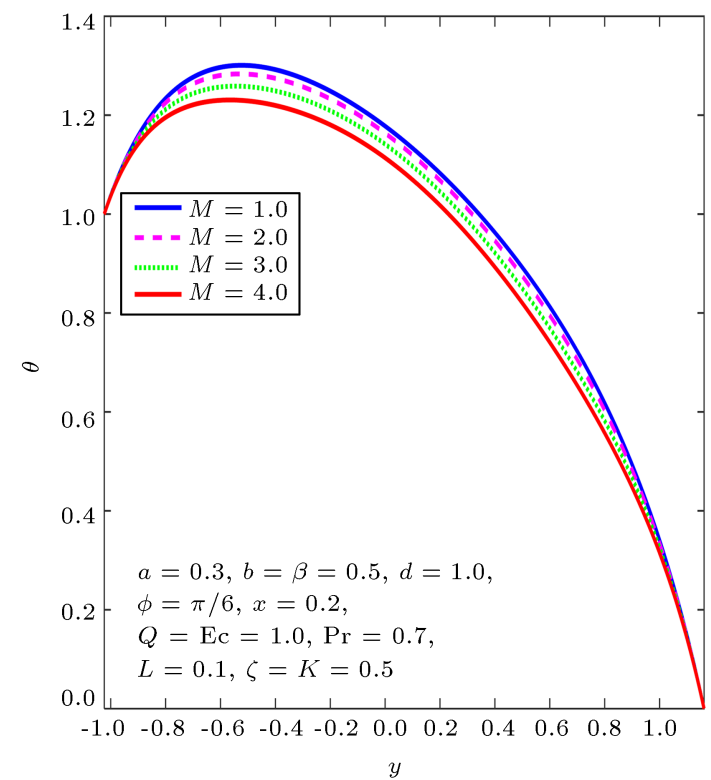

Figure 6. Impact of $M$ on $\theta(y)$.

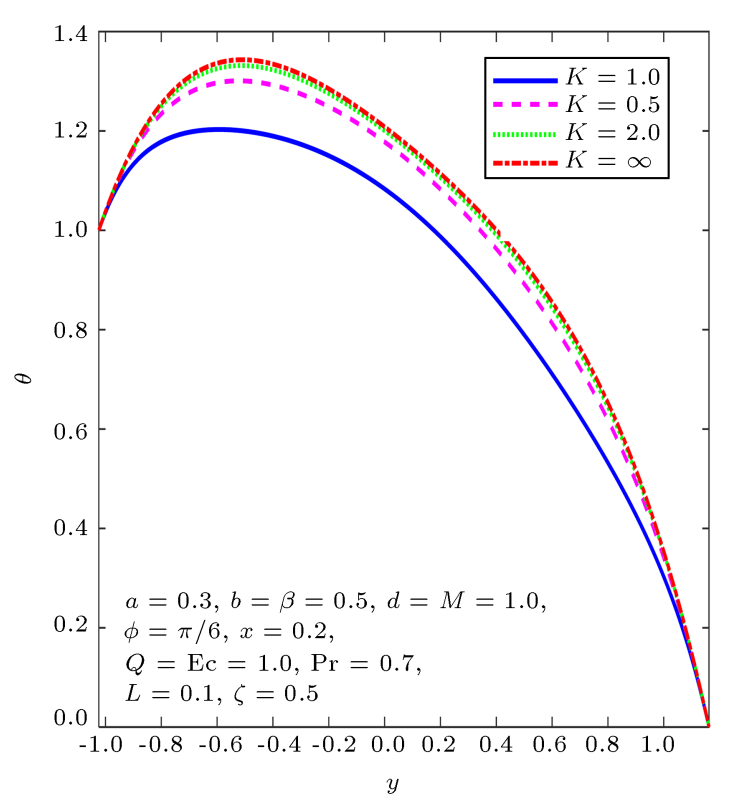

Figure 7. Impact of $K$ on $\theta(y)$.

permeability parameter. When fluid moves through a porous medium, internal energy is dissipated in the form of heat due to friction between fluid and the porous medium. Such energy loss is smaller when the porous medium possesses larger permeability. To perceive the behavior of Casson fluid parameter $\zeta$, the temperature distribution is presented in Figure 8. This figure shows that the temperature field decreases due to increase in the value of Casson fluid parameter. The influence of heat source parameter, $\beta$, on $\theta(y)$ is presented in Figure 9. It can be found that the dimensionless fluid temperature rises as the value of the heat source parameter, $\beta$, escalates. Furthermore, it is 


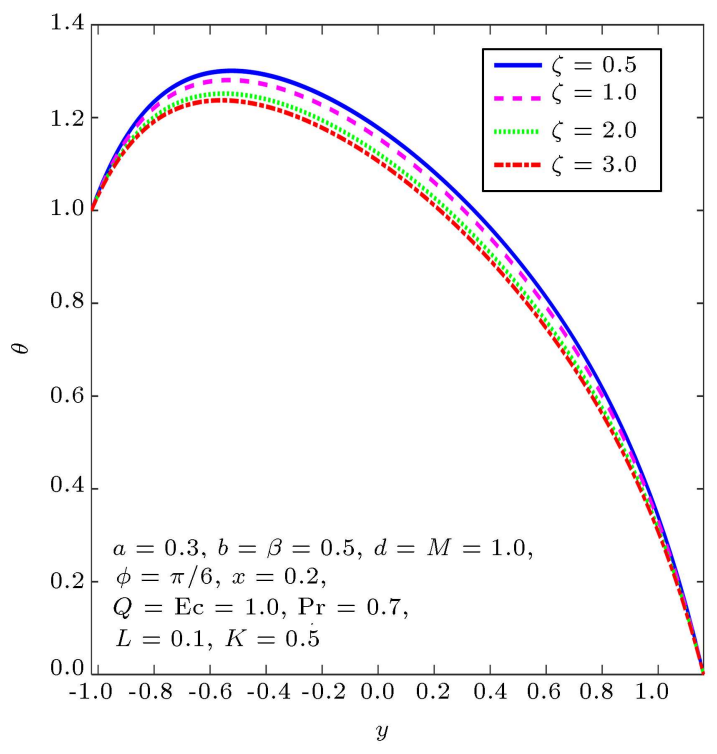

Figure 8. Impact of $\zeta$ on $\theta(y)$.

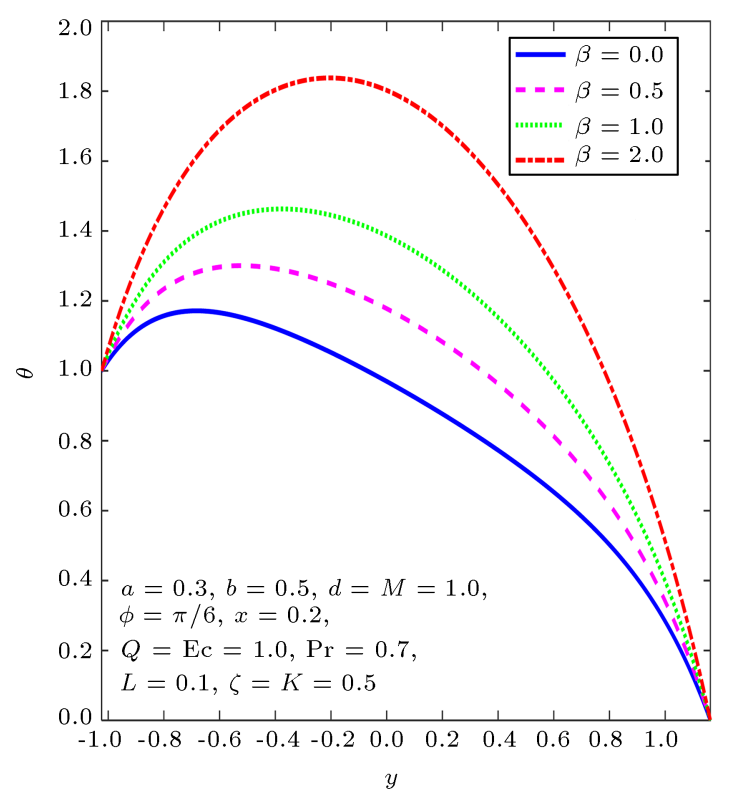

Figure 9. Impact of $\beta$ on $\theta(y)$.

noteworthy that the energy boundary layer thickness rises with increase in the value of $\beta$.

The axial pressure gradient for various values of $M, K, \zeta$, and $\phi$ are presented in Figures 10-13. In Figure 10, it can be observed that the pressure gradient increases with increase in $M$ and pressure gradient when MHD fluid is greater than hydrodynamic fluid. In Figure 11, the variation in pressure gradient along axial distance due to the influence of permeability parameter $K$ can be observed. It is found that the value of pressure gradient slightly decreases when permeability parameter rises in the central part of the channel. The pressure gradient for different values of the Casson fluid parameter, $\zeta$, is shown in Figure 12.

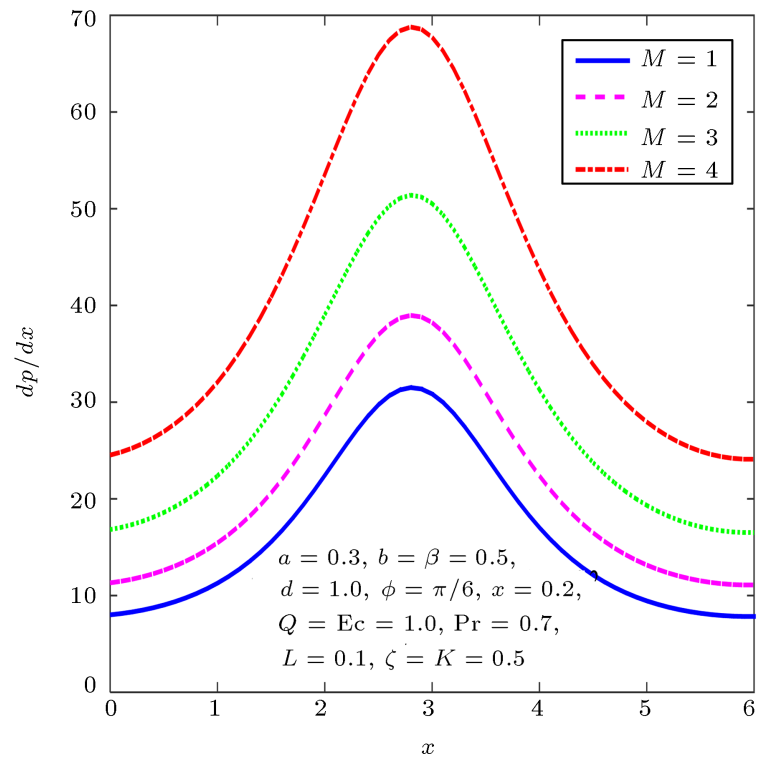

Figure 10. Impact of $M$ on pressure gradient.

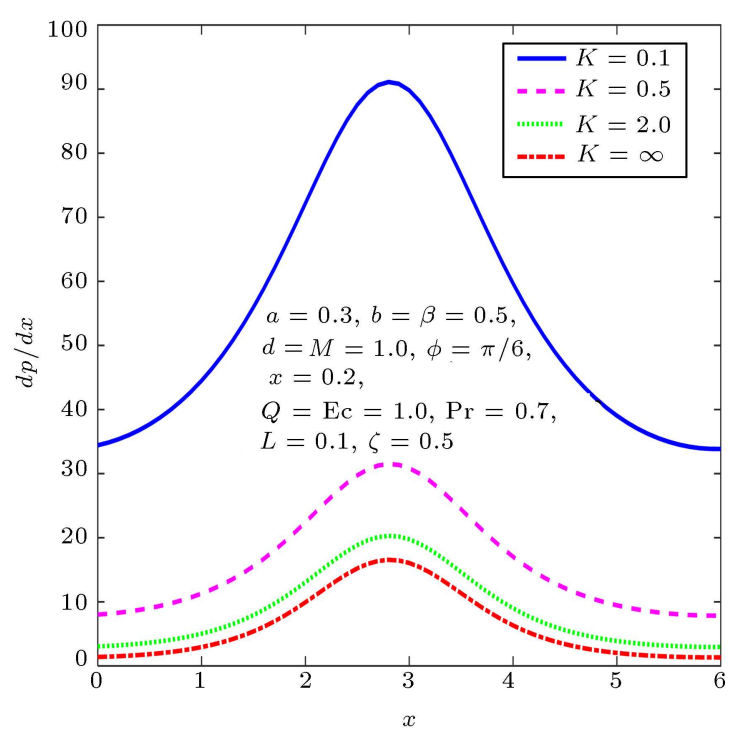

Figure 11. Impact of $K$ on pressure gradient.

The magnitude of pressure gradient is reduced with an increase in $\zeta$. Figure 13 depicts that the amplitude of the pressure gradient decreases with increase in $\phi$ and the point of maximum decreases with increase in $\phi$. The pressure rise (i.e., $\Delta p$ versus $Q$ ) for different values of the governing physical parameters is plotted in Figures 14-16. It is found that the pressure rise increases with increase in Hartmann number, $M$, and $\phi$ (see Figure 14). From Figures 15 and 16, it is found that in the pumping region, the pressure rise decreases with increase in Casson fluid parameter $\zeta$ and permeability parameter $K$, because the rise in viscosity and amplitude becomes slow and pressure rise decreases.

Figures 17-20 are presented to examine the effect 


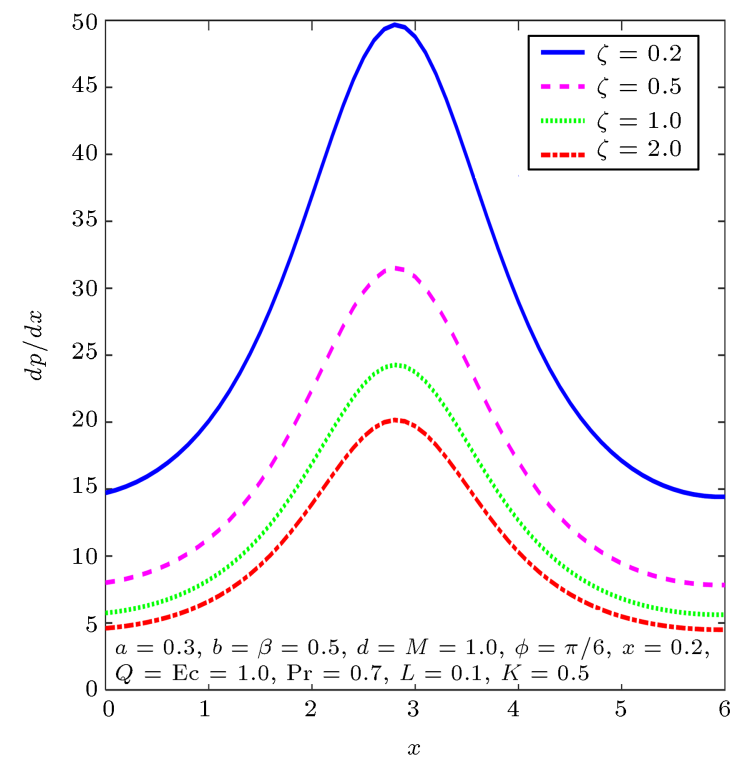

Figure 12. Impact of $\zeta$ on pressure gradient.

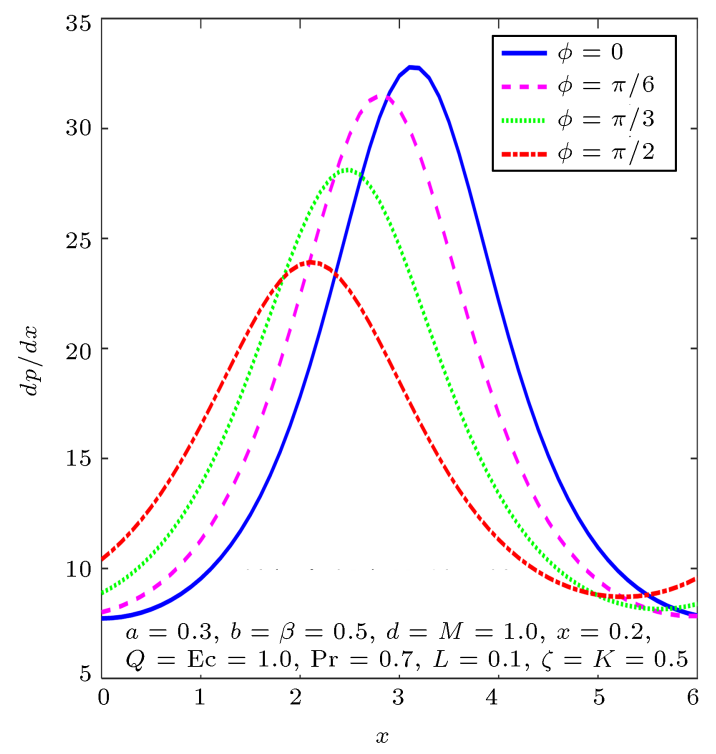

Figure 13. Impact of $\phi$ on pressure gradient.

of different values of the flow parameters on trapping. Figure 17 shows that the trapped bolus is less in size for the hydromagnetic fluid. In fact, the hydromagnetic characteristic rises because of the applied magnetic field. Consequently, the Lorentz force acts as a retarding force. This helps in reducing the size of trapped bolus. Figure 18 illustrates the effect of the permeability parameter $K$ on the streamlines for fixed values of other parameters. It is observed that the size of trapped bolus increases with increase in $K$. Figure 19 reveals that with an increase in Casson fluid parameter $\zeta$, trapping bolus decreases in the lower and upper parts of the channel. Figure 20 illustrates the effect of $\phi$ on trapping. It can be noticed that the

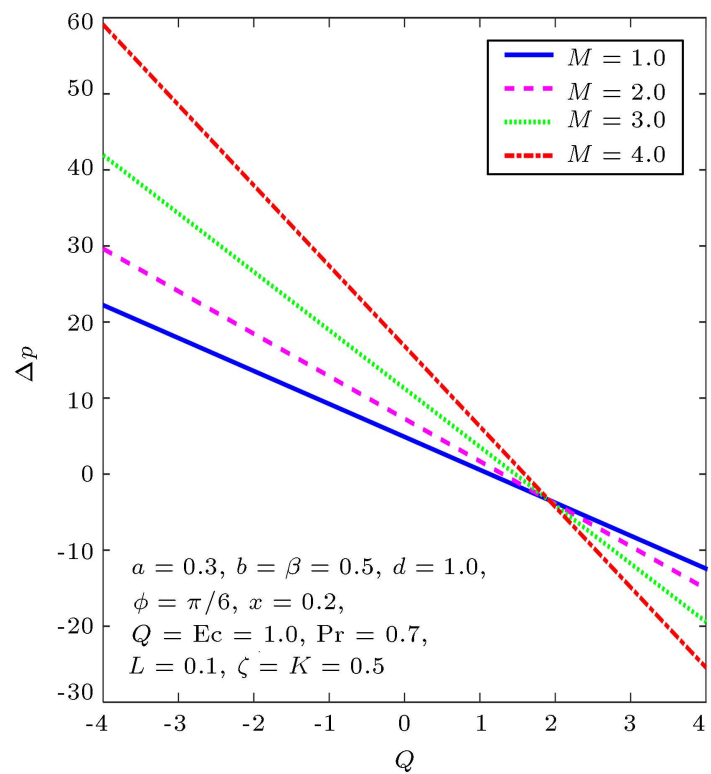

Figure 14. Impact of $M$ on pressure rise.

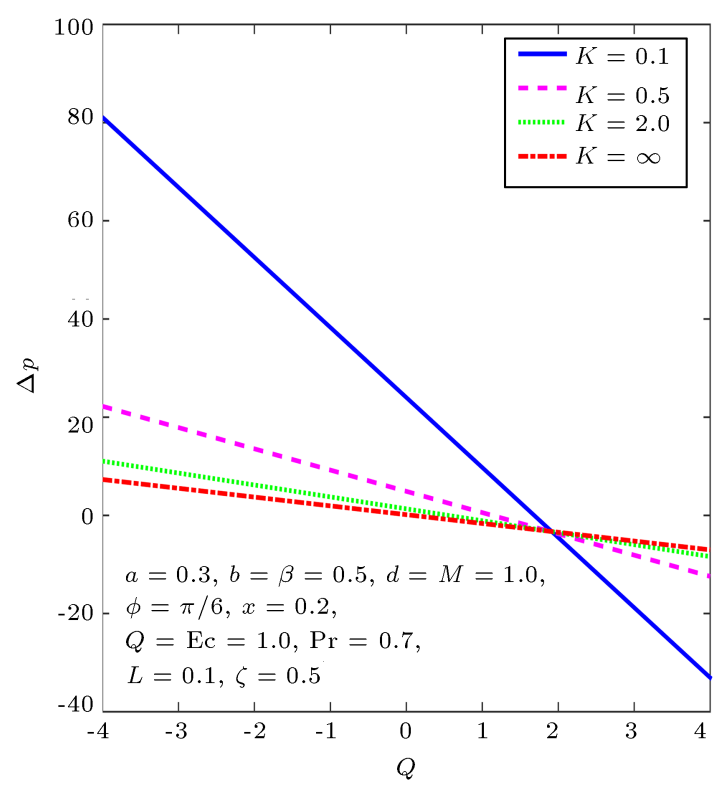

Figure 15. Impact of $K$ on pressure rise.

bolus appearing in the central region for $\phi=0$ moves towards left and decreases in size as $\phi$ increases.

Comparison of the present solution in a limited case of the obtained results with the ones in the published literature for some particular values of the governing parameters is shown in Table 1. Good agreement is observed between the present study and the existing literature. The influences of various flow parameters on the skin friction coefficient and Nusselt number at the lower and upper walls are displayed in Tables 2 and 3. Table 2 displays the variation in skin friction coefficient at the walls for various values of governing parameters of the flow. It is evident that 


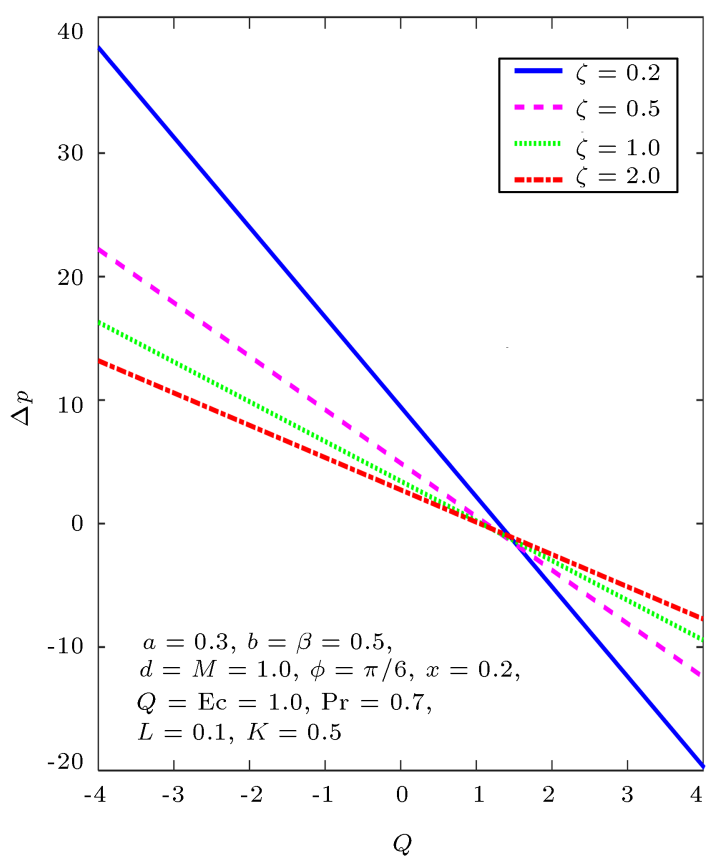

Figure 16. Impact of $\zeta$ on pressure rise.

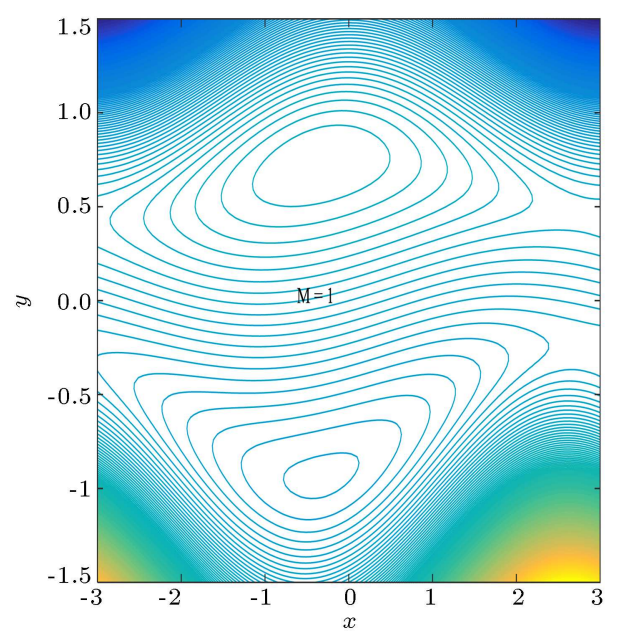

Table 1. Comparison of the values of pressure rise for the volume flow rate when $\zeta \rightarrow \infty$ and $L=0.1$.

\begin{tabular}{cccc}
\hline $\boldsymbol{Q}$ & Ebaid [16] & $\begin{array}{c}\text { Nadeem and } \\
\text { Akram [43] }\end{array}$ & $\begin{array}{c}\text { Present } \\
\text { results }\end{array}$ \\
\hline-2.0 & 5.98235 & 5.98235 & 5.98235 \\
-1.0 & 4.01392 & 4.01392 & 4.01392 \\
0.0 & 2.04548 & 2.04548 & 2.04548 \\
1.0 & 0.07704 & 0.07704 & 0.07704 \\
2.0 & -1.89139 & -1.89139 & -1.89139 \\
\hline
\end{tabular}

the magnitude of the skin friction coefficient rises with increase in the Hartmann number, while it is reduced with increase in the slip parameter, permeability parameter, and Casson fluid parameter. The variation in Nusselt number at the walls for different values of the governing flow parameters is depicted in Table 3. From this table, it is found that the magnitude of Nusselt number at the lower and upper walls is enhanced with

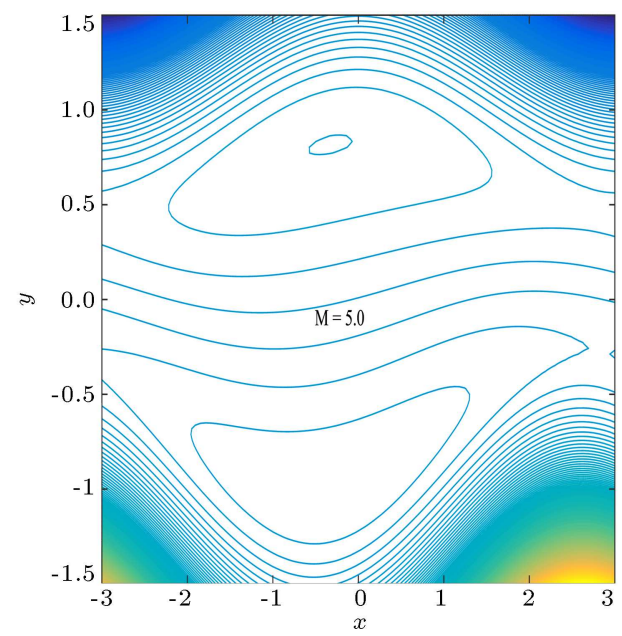

Figure 17. Streamlines for different values of $M$.
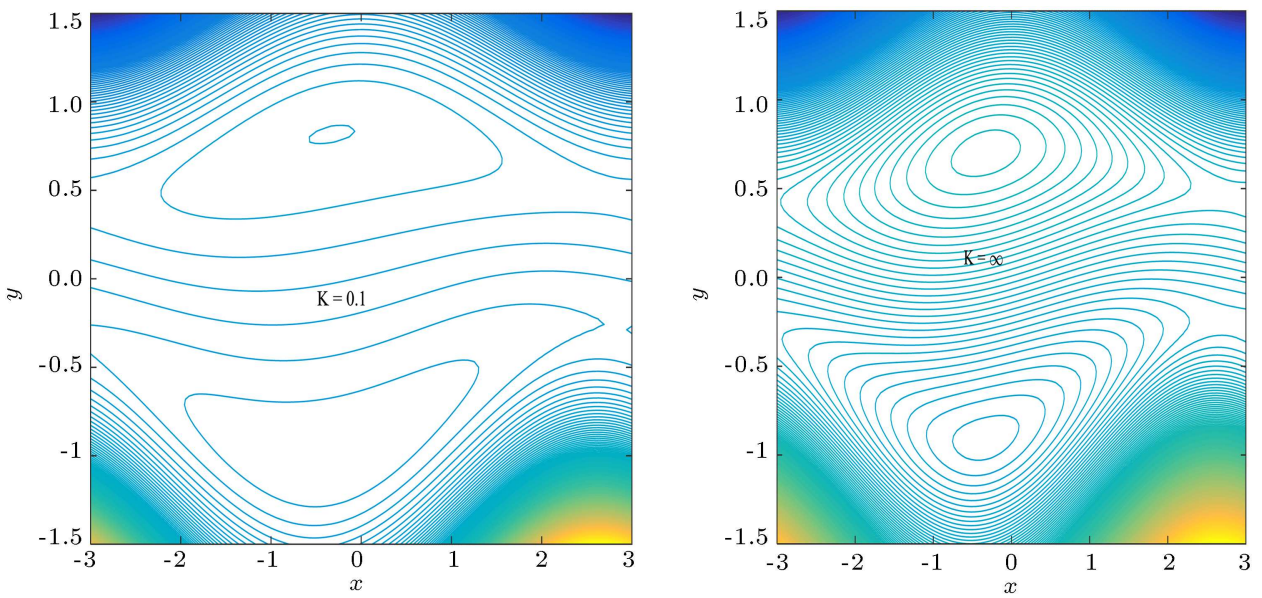

Figure 18. Streamlines for different values of $K$. 

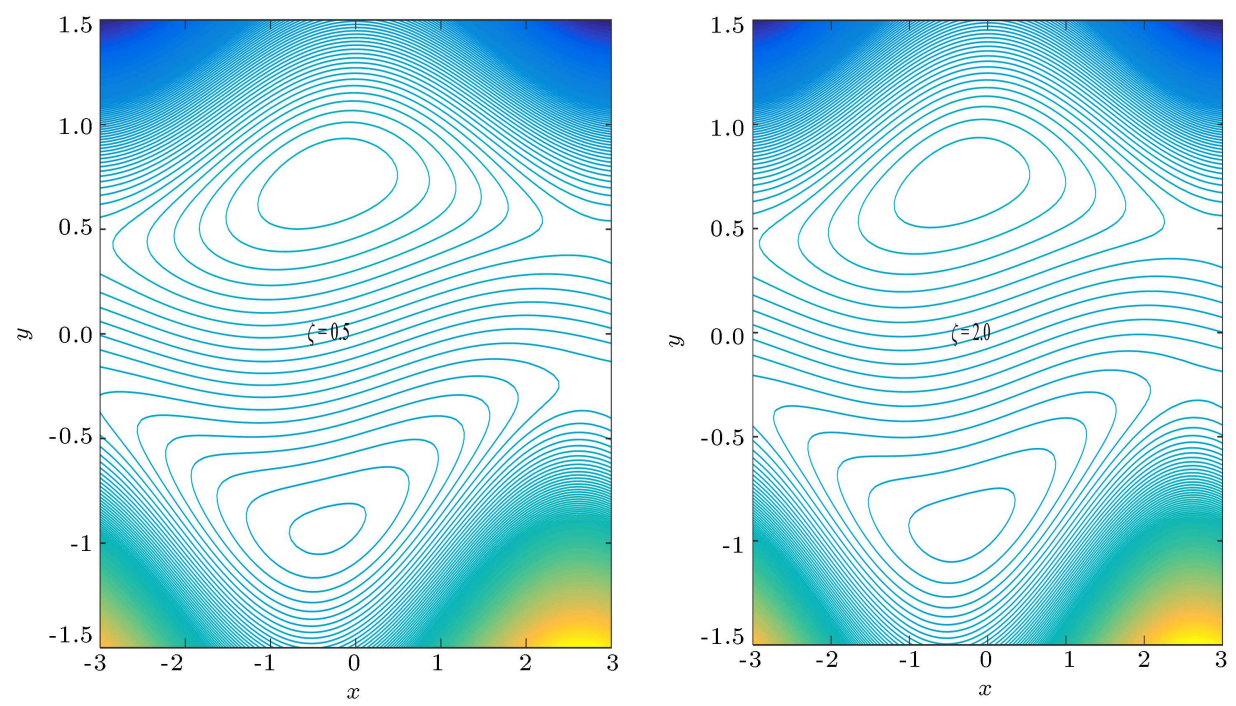

Figure 19. Streamlines for different values of $\zeta$.
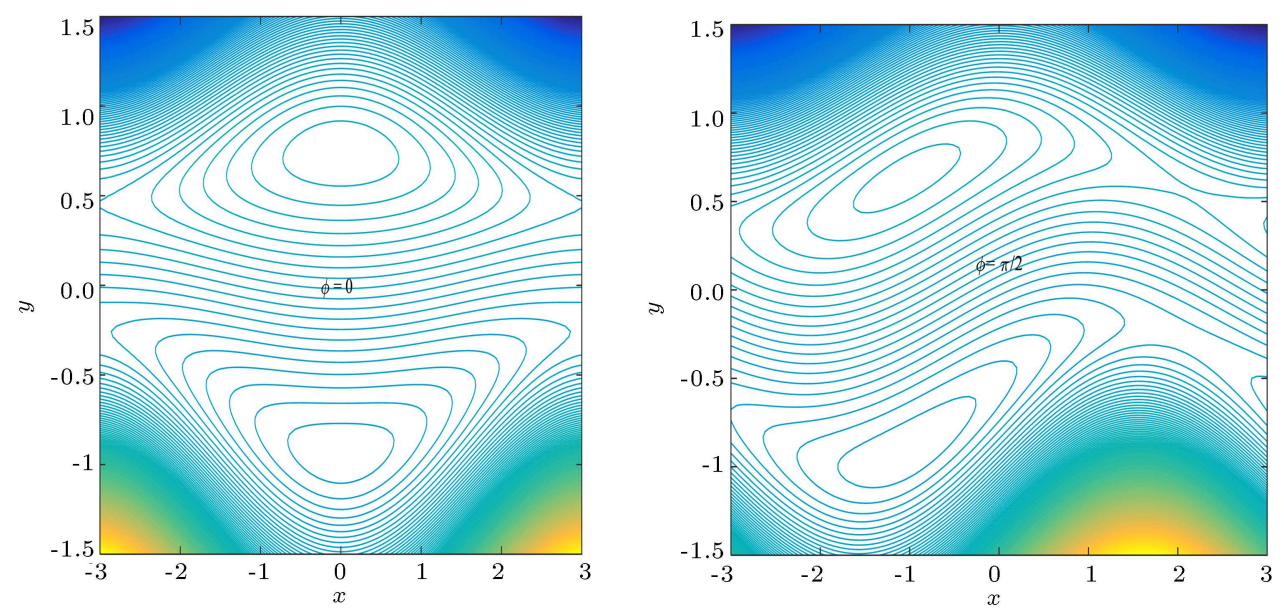

Figure 20. Streamlines for different values of $\phi$.

Table 2. Variation in skin-friction coefficient, $C_{f}$, at the wall for different governing non-dimensional flow parameters.

\begin{tabular}{cccccc}
\hline $\boldsymbol{L}$ & $\boldsymbol{M}$ & $\boldsymbol{K}$ & $\boldsymbol{\zeta}$ & $\left.\boldsymbol{C}_{\boldsymbol{f}}\right|_{\boldsymbol{y}=h_{\boldsymbol{1}}}$ & $\left.\boldsymbol{C}_{\boldsymbol{f}}\right|_{\boldsymbol{y}=h_{2}}$ \\
\hline 0.1 & 1.0 & 0.5 & 0.5 & -2.8593 & 2.8593 \\
0.2 & & & & -1.9621 & 1.9621 \\
0.3 & & & & -1.4934 & 1.4934 \\
& 2.0 & & & -2.9712 & 2.9712 \\
& 3.0 & & & -3.1284 & 3.1284 \\
& & \multirow{2}{*}{1.0} & & -2.7295 & 2.7295 \\
& & & -2.6304 & 2.6304 \\
& & & 1.0 & -2.2673 & 2.2673 \\
& & & 2.0 & -1.8863 & 1.8863 \\
\hline
\end{tabular}

increase in the Hartmann number, Prandtl number, and heat source parameter, while it diminishes with increase in the slip, permeability, and Casson fluid parameters.

\section{Conclusions}

The heat transfer analysis of magnetohydrodynamic peristaltic Casson fluid flow filled with a porous medium in asymmetric channel in presence of partial slip was presented. The interaction of various governing flow parameters with peristaltic Casson fluid flow was also analyzed. The key findings of this work are summarized as follows:

- The axial velocity increased with increase in slip parameter, $L$, and Hartmann number, $M$, near the channel walls, while velocity decreased at the center of the channel; the opposite behavior was observed in the permeability parameter, $K$, and Casson fluid parameter $\zeta$;

- Temperature distribution was reduced with increase in $M$ and $\zeta$, while the temperature profile was enhanced with the rise of $K$ and $\beta$;

- It was observed that an increase in the slip param- 
Table 3. Variation in Nusselt Number $\mathrm{Nu}$ at the lower and upper walls for different governing non-dimensional flow parameters.

\begin{tabular}{|c|c|c|c|c|c|c|c|}
\hline$L$ & $M$ & $K$ & $\zeta$ & $\operatorname{Pr}$ & $\beta$ & $\left.\mathbf{N u}\right|_{y=h_{1}}$ & $\left.\mathrm{Nu}\right|_{y=h_{2}}$ \\
\hline 0.1 & 1.0 & 0.5 & 0.5 & 0.7 & 0.5 & 2.4390 & -1.6896 \\
\hline 0.2 & & & & & & 2.0796 & -1.3302 \\
\hline \multirow[t]{11}{*}{0.3} & & & & & & 1.9451 & -1.1957 \\
\hline & 2.0 & & & & & 2.8358 & -2.0863 \\
\hline & 3.0 & & & & & 3.4932 & -2.7438 \\
\hline & & 1.0 & & & & 2.0395 & -1.2901 \\
\hline & & 3.0 & & & & 1.7710 & -1.0216 \\
\hline & & & 1.0 & & & 2.1300 & -1.3806 \\
\hline & & & 2.0 & & & 1.9429 & -1.1935 \\
\hline & & & & 1.0 & & 3.3237 & -2.5743 \\
\hline & & & & 2.0 & & 6.2727 & -5.5233 \\
\hline & & & & & 1.0 & 2.9060 & -2.1566 \\
\hline & & & & & 2.0 & 3.8401 & -3.0907 \\
\hline
\end{tabular}

eter would lead to decrease in the magnitude of the pressure gradient, and the magnitude of pressure gradient decreased with an increase in $\zeta$;

- The pressure rise increased with increase in Hartmann number, $M$, and amplitude, $\phi$;

- The size of trapped bolus increased with increase in $K$;

- The magnitude of Nusselt number at the lower and upper walls was enhanced with increase in the Hartmann number and heat source parameter, while it diminished with increase in the permeability parameter and Casson fluid parameter.

\section{Acknowledgement}

The authors appreciate the valuable suggestions of the anonymous reviewers towards the improvement of the manuscript. This work was partly supported by National Research Foundation of South Africa.

\section{Nomenclature}

$\begin{array}{ll}a_{1}, b_{1} & \text { Amplitudes of the waves } \\ c & \text { Velocity of propagation } \\ C_{f} & \text { Skin-friction coefficient } \\ d_{1}+d_{2} & \text { Width of the channel }\end{array}$

$u$ and $v \quad$ Velocity components in the wave frame $(x, y)$

$X \quad$ Direction of wave propagation

$(x, y) \quad$ Wave frame

$(X, Y) \quad$ Laboratory frame

$Y \quad$ Perpendicular to $X$

\section{Greek symbols}

$\beta \quad$ Heat source parameter

$\lambda \quad$ Wave length 
$\phi \quad$ Phase difference

$\pi_{c} \quad$ A critical value of $\pi$

$\mu_{B} \quad$ Plastic viscosity of the fluid

$\zeta \quad$ Casson fluid parameter

\section{References}

1. Mishra, M. and Rao, A.R. "Peristaltic transport of a Newtonian fluid in an asymmetric channel", Zeitschriftfür Angewandte Mathematik und Physik, 54, pp. 532-550 (2003).

2. Ali, N., Hayat, T., and Sajid, M. "Peristaltic flow of a couple stress fluid in asymmetric channel", Biorheology, 44, pp. 125-138 (2007).

3. Subba Reddy, M.V., Rao, A.R., and Sreenath, S. "Peristaltic motion of a power-law fluid in an asymmetric channel", International Journal of Non-Linear Mechanics, 42, pp. 1153-1161 (2007).

4. Kothandapani, M. and Srinivas, S. "Non-linear peristaltic transport of Newtonian fluid in an inclined asymmetric channel through a porous medium", Physics Letters A, 372, pp. 1265-1276 (2008).

5. Makinde, O.D. and Mhone, P.Y. "Heat transfer to MHD oscillatory flow in a channel filled with porous medium", Romanian Journal of Physics, 50(9/10), pp. 931-938 (2005).

6. Makinde, O.D. "Collapsible tube flow-A mathematical model", Romanian Journal of Physics, 50(5/6), pp. 493-506 (2005).

7. Makinde, O.D. "Asymptotic approximations for oscillatory flow in a tube of varying cross-section with permeable isothermal wall", Romanian Journal of Physics, 52(1/2), pp. 59-72 (2007).

8. Stud, V.K., Sephon, G.S., and Mishra, R.K. "Pumping action on blood flow by a magnetic field", Bull. Math. Biol., 39, pp. 385-390 (1977).

9. Srivastava, L.M. and Agrawal, R.P. "Oscillating flow of a conducting fluid with a suspension of spherical particles", J. Appl. Mech., 47, pp. 196-200 (1980).

10. Agrawal, H.L. and Anwaruddin, B. "Peristaltic flow of blood in a branch", Ranchi Univ. Math. J., 15, pp. 111-121 (1984).

11. Wang, Y., Hayat, T., and Hutter, K. "On non-linear magnetohydrodynamics problems of an Oldroyd-6constant fluid", International Journal of Non-linear Mechanics, 40, pp. 40-58 (2005).

12. Nadeem, S. and Akbar, N.S. "Effects of heat transfer on the peristaltic transport of MHD Newtonian fluid with variable viscosity: application of Adomian de- composition method", Communications in Nonlinear Science and Numerical Simulation, 14, pp. 3844-3855 (2009).

13. Ikbal, M.d.A., Chakravarty, S., Wong, K.K.L., Mazumdar, J., and Mandal, P.K. "Unsteady response of non-Newtonian blood flow through a stenosed artery in magnetic field", Journal of Computational and Applied Mathematics, 230, pp. 243-259 (2009).

14. Gnaneswara Reddy, M. "Velocity and thermal slip effects on MHD third order blood flow in an irregular channel though a porous medium with homogeneous / heterogeneous reactions", Nonlinear Engineering, 6(3), pp. 167-178 (2017). DOI: https://doi.org/10.1515/nleng-2017-0008

15. Sarkar, B.C., Das, S., Jana, R.N., and Makinde, O.D. "Magnetohydrodynamic peristaltic flow on nanofluids in a convectively heated vertical asymmetric channel in the presence of thermal radiation", Journal of Nanofluids, 4, pp. 1-10 (2015).

16. Ebaid, A. "Effects of magnetic field and wall slip conditions on the peristaltic transport of a Newtonian fluid in an asymmetric channel", Physics Letters A, 372, pp. 4493-4499 (2008).

17. Akbar, N.S., Hayat, T., Nadeem, S., and Hendi, A.A. "Effects of slip and heat transfer on the peristaltic flow of a third order fluid in an inclined asymmetric channel", International Journal of Heat and Mass Transfer, 54, pp. 1654-1664 (2011).

18. Makinde, O.D. and Osalusi, E. "MHD steady flow with slip in a channel with permeable boundaries", Romanian Journal of Physics, 51(3/4), pp. 293-302 (2006).

19. Nadeem, S. and Akram, S. "Slip effects on the peristaltic flow of a Jeffrey fluid in an asymmetric channel under the effect of induced magnetic field", International Journal for Numerical Methods in Fluids, 63, pp. 374-394 (2010).

20. Hayat, T., Quereshi, M.U., and Hussain, Q. "Effect of heat transfer on the peristaltic flow of an electrically conducting fluid in a porous space", Appl. Math. Model, 33, pp. 1862-1873 (2009).

21. Makinde, O.D. and Mhone, P.Y. "Hydromagnetic effects on internal flow separation in a diverging channel", Romanian Journal of Physics, 51(9/10), pp. 959966 (2006).

22. Radhakrishnamacharya, G. and Srinivasulu, C. "Influence of wall properties on peristaltic transport with heat transfer", CR Mec., 335, pp. 369-373 (2007).

23. Srinivas, S. and Kothandapani, M. "Peristaltic transport in an asymmetric channel with heat transfer-a note", Int. Commun Heat Mass Transfer, 35, pp. 514$522(2008)$. 
24. Srinivas, S., Gayathri, R., and Kothandapani, M. "The influence of slip conditions, wall properties and heat transfer on MHD peristaltic transport", Comput. Phys. Commun., 180, pp. 2115-2122 (2009).

25. Mekheimer, K.S. and Abd Elmaboud, Y. "The influence of heat transfer and magnetic field on peristaltic transport of a Newtonian fluid in a vertical annulus: application of an endoscope", Phys Lett A, 372, pp. 1657-1665 (2008).

26. Gnaneswara Reddy, M. and Venugopal Reddy, K. "Influence of Joule heating on MHD peristaltic flow of a nanofluid with compliant walls", Procedia Engineering, 127, pp. 1002-1009 (2015).

27. Gnaneswara Reddy, M. "Heat and mass transfer on magnetohydrodynamic peristaltic flow in a porous medium with partial slip", Alexandria Engineering Journal, 55, pp. 1225-1234 (2016).

28. Gnaneswara Reddy, M., Venugopal Reddy, K., and Makinde, O.D. "Hydromagnetic peristaltic motion of a reacting and radiating couple stress fluid in an inclined asymmetric channel filled with a porous medium", Alexandria Engineering Journal, 55, pp. 1841-1853 (2016).

29. Srinivas, S. and Muthuraj, R. "Effects of chemical reaction and space porosity on MHD mixed convective flow in a vertical asymmetric channel with peristalsis", Mathematical and Computer Modelling, 54(5/6), pp. 1213-1227 (2011).

30. Akram, S. and Nadeem, S. "Influence of induced magnetic field and heat transfer on the peristaltic motion of a Jeffrey fluid in an asymmetric channel: Closed form solutions", Journal of Magnetism and Magnetic Materials, 328, pp. 11-20 (2013).

31. Gnaneswara Reddy, M., Venugopal Reddy, K., and Makinde, O.D. "Heat transfer on MHD peristaltic rotating flow of a Jeffrey fluid in an asymmetric channel", International Journal of Applied and Computational Mathematics, 3(4), pp. 3201-3227 (2017). DOI:10.1007/s40819-016-0293-1

32. Gnaneswara Reddy, M. and Makinde, O.D. "Magnetohydrodynamic peristaltic transport of Jeffery nanofluid in an asymmetric channel", Journal of Molecular Liquids, 223, pp. 1242-1248 (2016).

33. Ellahi, R., Fetecau, C., and Vafai, K. "Peristaltic flow of couple stress fluid in a non-uniform rectangular duct having compliant walls", Communications in Theoretical Physics, 65(1), pp.66-72 (2016).

34. Bhatti, M.M., Zeeshan, A., and Ellahi, R. "Heat transfer analysis on peristaltically induced motion of particle-fluid suspension with variable viscosity: Clot blood model", Computer Methods and Programs in Biomedicine, 137, pp. 115-124 (2016).

35. Ellahi, R., Bhatti, M.M., and Pop, I. "Effects of hall and ion slip on MHD peristaltic flow of Jeffrey fluid in a non-uniform rectangular duct", International Journal of Numerical Methods for Heat \& Fluid Flow, 26(6), pp. 1802-1820 (2016).

36. Bhatti, M.M., Ellahi, R., and Zeeshan, A. " Study of variable magnetic field on the peristaltic flow of Jeffrey fluid in a non-uniform rectangular duct having compliant walls", Journal of Molecular Liquids, 222, pp. 101-108 (2016).

37. Bhatti, M.M., Zeeshan, A., and Ellahi, R. "Simultaneous effects of coagulation and variable magnetic field on peristaltically induced motion of Jeffrey nanofluid containing gyrotactic microorganism", Microvascular Research, 110, pp. 32-42 (2017).

38. Bhatti, M.M., Zeeshan, A., Ellahi, R., and Ijaz, N. "Heat and mass transfer of two-phase flow with electric double layer effects induced due to peristaltic propulsion in the presence of transverse magnetic field", Journal of Molecular Liquids, 230, pp. 237-246 (2017).

39. Bhatti, M.M., Zeeshan, A., and Ellahi, R. "Endoscope analysis on peristaltic blood flow of Sisko fluid with Titanium magneto-nanoparticles", Computers in Biology and Medicine, 78, pp. 29-41 (2016).

40. Ellahi, R., Bhatti, M.M., Pop, I., and Vafai, K. "Effects of heat and mass transfer on peristaltic flow in a nonuniform rectangular duct", International Journal of Heat and Mass Transfer, 71, pp. 706-719 (2014).

41. Casson, N. "Flow equation for pigment oil suspensions of the printing ink type", Rheology of Disperse Systems, C.C. Mill, Ed., Pergamon Press, London, UK, pp. 84-102 (1959).

42. Akbar, N.S. "Influence of magnetic field on peristaltic flow of a Casson fluid in an asymmetric channel: Application in crude oil refinement", Journal of Magnetism and Magnetic Materials, 378, pp. 463-468 (2015).

43. Nadeem, S. and Akram, S. "Heat transfer in a peristaltic flow of MHD fluid with partial slip", Commun Nonlinear Sci Numer Simulat, 15, pp. 312-321 (2010).

44. Ijaz, N., Bhatti, M.M., and Zeeshan, A. "Heat transfer analysis in MHD flow of solid particles in nonNewtonian Ree-Eyring fluid due to peristaltic wave in a channel", Thermal Science, 23(2B), pp. 1017-1026 (2019).

45. Srinivas, S. and Muthuraj, R. "Peristaltic transport of a Jeffrey fluid under the effect of slip in an inclined asymmetric channel", Int. J. Appl. Mechanics, 2, pp. 437-455 (2010).

\section{Appendix}

The unknowns involved in Eqs. (16) and (17) are defined in Box A.I. 


$$
\begin{aligned}
& N^{2}=\left(\frac{\zeta}{1+\zeta}\right)\left[M^{2}+\frac{1}{K}\right], \quad N_{1}=L\left(1+\frac{1}{\zeta}\right), \quad A=\operatorname{Pr} \operatorname{Ec}\left(1+\frac{1}{\zeta}\right), \\
& B=\operatorname{Pr}\left[M^{2}+\left(1+\frac{1}{\zeta}\right) \frac{1}{K}\right], \quad C=\operatorname{Pr} \beta \\
& F_{0}=\frac{\left(h_{1}+h_{2}\right)\left[N q \cosh \left(\frac{1}{2}\left(h_{1}-h_{2}\right) N\right)+\left(2+N_{1} N^{2} q\right) \sinh \left(\frac{1}{2}\left(h_{1}-h_{2}\right) N\right)\right]}{2\left(-\left(h_{1}-h_{2}\right) N \cosh \left(\frac{1}{2}\left(h_{1}-h_{2}\right) N\right)+\left(2+A\left(-h_{1}+h_{2}\right) N^{2}\right) \sinh \left(\frac{1}{2}\left(h_{1}-h_{2}\right) N\right)\right.}, \\
& F_{1}=\frac{N q \cosh \left(\frac{1}{2}\left(h_{1}-h_{2}\right) N_{1}\right)+\left(2+N_{1} N^{2} q\right) \sinh \left(\frac{1}{2}\left(h_{1}-h_{2}\right) N\right)}{\left(h_{1}-h_{2}\right) N \cosh \left(\frac{1}{2}\left(h_{1}-h_{2}\right) N_{1}\right)+\left(-2+N_{1}\left(h_{1}-h_{2}\right) N^{2}\right) \sinh \left(\frac{1}{2}\left(h_{1}-h_{2}\right) N\right)} \\
& F_{2}=\frac{\left(h_{1}-h_{2}+q\right)\left(\cosh \left(h_{1} N\right)-\cosh \left(h_{2} N\right)+N N_{1}\left(\sinh \left(h_{1} N\right)+\sinh \left(h_{2} N\right)\right)\right)}{2\left(N N_{1} \cosh \left(\frac{1}{2}\left(h_{1}-h_{2}\right) N\right)+\sinh \left(\frac{1}{2}\left(h_{1}-h_{2}\right) N\right)\right)\left(\begin{array}{c}
\left(h_{1}-h_{2}\right) N \cosh \left(\frac{1}{2}\left(h_{1}-h_{2}\right) N\right) \\
+\left(-2+N_{1}\left(h_{1}-h_{2}\right) N^{2}\right)+\sinh \left(\frac{1}{2}\left(h_{1}-h_{2}\right) N\right)
\end{array}\right)}, \\
& F_{3}=\frac{\left(h_{1}-h_{2}+q\right) \cosh \left(\frac{1}{2}\left(h_{1}+h_{2}\right) N\right)}{\left(h_{1}-h_{2}\right) N \cosh \left(\frac{1}{2}\left(h_{1}-h_{2}\right) N\right)+\left(-2+N_{1}\left(h_{1}-h_{2}\right) N^{2}\right) \sinh \left(\frac{1}{2}\left(h_{1}-h_{2}\right) N\right)}, \\
& c_{1}=-\frac{1}{8\left(h_{1}-h_{2}\right) N} \\
& \left.h_{2}\left[\begin{array}{l}
16 B F_{1} F_{3} \cosh \left(h_{1} N\right)+\left(\mathrm{f}_{2}^{2}+F_{3}^{2}\right) N\left(B+N_{1} N^{2}\right) \cosh \left(2 h_{1} N\right) \\
+2\left(8 B F_{1} F_{2} \sinh \left(h_{1} N\right)+N\left(h_{1}^{2}\left[\begin{array}{l}
2 C+N_{1}\left(F_{2}^{2}-F_{3}^{2}\right) N^{4} \\
+B\left(2 F_{1}^{2}+\left(F_{3}^{2}-F_{2}^{2}\right) N^{2}\right) \\
+F_{2} F_{3}\left(B+N_{1} N^{2}\right) \sinh \left(2 h_{1} N\right)
\end{array}\right]\right)\right.
\end{array}\right]\right) \\
& \left.-h_{1}\left[\begin{array}{l}
16 B F_{1} F_{3} \cosh \left(h_{2} N\right)+\left(\mathrm{f}_{2}^{2}+F_{3}^{2}\right) N\left(B+N_{1} N^{2}\right) \cosh \left(2 h_{2} N\right) \\
\left.+2\left(\begin{array}{l}
8 F_{1} F_{2} \sinh \left(h_{2} N\right)+N \\
+B F_{3}^{2} h_{2}^{2} N^{2}+N_{1} F_{2}^{2} h_{2}^{2} N^{4}-N_{1} F_{3}^{2} h_{2}^{2} N^{4} \\
+F_{2} F_{3}\left(B+N_{1} N^{2}\right) \sinh \left(2 h_{2} N\right)
\end{array}\right]\right)
\end{array}\right]\right) \\
& c_{2}=-\frac{1}{8\left(h_{1}-h_{2}\right) N} \\
& {\left[\begin{array}{l}
8 N-4 C h_{1}^{2} N-4 B F_{1}^{2} h_{1}^{2} N+4 C h_{2}^{2} N+4 B F_{1}^{2} h_{2}^{2} N+2 B F_{2}^{2} h_{1}^{2} N^{3}-2 B F_{3}^{2} h_{1}^{2} N^{3} \\
-2 B F_{2}^{2} h_{2}^{2} N^{3}+2 B F_{3}^{2} h_{2}^{2} N^{3}-2 A F_{2}^{2} h_{1}^{2} N^{5}+2 A F_{3}^{2} h_{1}^{2} N^{5}+2 A F_{2}^{2} h_{2}^{2} N^{5} \\
-2 A F_{3}^{2} h_{2}^{2} N^{5}-16 B F_{1} F_{3} \cosh \left(h_{1} N\right)-\left(F_{2}^{2}+F_{3}^{2}\right) N\left(B+N_{1} N^{2}\right) \cosh \left(2 h_{1} N\right)
\end{array}\right.} \\
& +16 B F_{1} F_{3} \cosh \left(h_{2} N\right)+B F_{2}^{2} N \cosh \left(2 h_{2} N\right)+B F_{3}^{2} N \cosh \left(2 h_{2} N\right) \\
& +N_{1} F_{2}^{2} N^{3} \cosh \left(2 h_{2} N\right)+N_{1} F_{3}^{2} N^{3} \cosh \left(2 h_{2} N\right)-16 B F_{1} F_{2} \sinh \left(h_{1} N\right) \\
& -2 B F_{3} F_{2} \sinh \left(2 h_{1} N\right)-2 N_{1} F_{2} F_{3} N^{3} \sinh \left(2 h_{1} N\right)+16 B F_{1} F_{2} \sinh \left(h_{2} N\right) \\
& +2 B F_{3} F_{2} \sinh \left(2 h_{2} N\right)+2 N_{1} F_{2} F_{3} N^{3} \sinh \left(2 h_{2} N\right)
\end{aligned}
$$




\section{Biographies}

Oluwole Daniel Makinde is a distinguished Professor of Applied Mathematics and Computations in the Faculty of Military Science, Stellenbosch University, South Africa. He is a fellow of African Academy of Sciences and Papua New Guinea Mathematical Society, and winner of African UnionKwame Nkrumah Continental Scientific Award 2011, NRF/NSTF TW-Kambule Outstanding Researcher A ward 2010 in South Africa, Nigerian National Honour Award (MFR), and several other research excellence awards. His research work covers a broad range of topics including mathematical modelling, heat and mass transfer, fluid mechanics, nanofluid dynamics, solid mechanics, bio-mathematics, combustion theory, etc.

Machireddy Gnaneswara Reddy is presently an Assistant Professor of Mathematics at the Acharya Nagarjuna University Campus, Ongole, India. He completed his $\mathrm{PhD}$ degree in Mathematics in 2009 at Sri Venkateswara University, India. His research interest is in the area of heat and mass transfer, porous media, magnetohydrodyanmics, and applied cryptography. He has published a number of research articles in reputable journals in these areas and guided some postgraduate students. 